\title{
Parallel Tempering: Theory, Applications, and New Perspectives
}

\author{
David J. Earl ${ }^{a, b}$ and Michael W. Deem ${ }^{a}$ \\ ${ }^{a}$ Departments of Bioengineering and Physics \& Astronomy, Rice University, \\ 6100 Main Street MS142, Houston, Texas 77005 USA. \\ E-mail: mwdeem@rice.edu \\ ${ }^{b}$ Rudolf Peierls Centre for Theoretical Physics, Oxford University, \\ 1 Keble Road, Oxford OX1 3NP United Kingdom. \\ E-mail: earl@thphys.ox.ac.uk
}

October 29, 2018

To appear in Physical Chemistry Chemical Physics

We review the history of the parallel tempering simulation method. From its origins in data analysis, the parallel tempering method has become a standard workhorse of physiochemical simulations. We discuss the theory behind the method and its various generalizations. We mention a selected set of the many applications that have become possible with the introduction of parallel tempering and we suggest several promising avenues for future research.

\section{Introduction}

The origins of the parallel tempering, or replica exchange, simulation technique can be traced to a 1986 paper by Swendsen and Wang. ${ }^{1}$ In this paper, a method of replica Monte Carlo was introduced in which replicas of a system of interest are simulated at a series of temperatures. Replicas at adjacent temperatures undergo a partial exchange of configuration information. The more familiar form of parallel tempering with complete exchange of configuration information was formulated by Geyer. The first description of the parallel tempering algorithm was found in the preprint of his 1991 manuscript, ${ }^{2}$ although only a simulated tempering approach appeared in the final publication, due to a technical 
problem with a related but inessential statistical theorem for standard errors. Geyer and Thompson later published the now widely recognized full paper describing the parallel tempering method in $1995 .^{3}$ Initially, applications of the new method were limited to problems in statistical physics. However, following Hansmann's use of the method in Monte Carlo simulations of a biomolecule, ${ }^{4}$ Falcioni and Deem's use of parallel tempering for X-ray structure determination, ${ }^{5}$ and Okamoto and co-worker's formulation of a molecular dynamics version of parallel tempering, ${ }^{6}$ the use of parallel tempering in fields spanning physics, chemistry, biology, engineering and materials science rapidly increased.

The general idea of parallel tempering is to simulate $M$ replicas of the original system of interest, each replica typically in the canonical ensemble, and usually each replica at a different temperature. The high temperature systems are generally able to sample large volumes of phase space, whereas low temperature systems, whilst having precise sampling in a local region of phase space, may become trapped in local energy minima during the timescale of a typical computer simulation. Parallel tempering achieves good sampling by allowing the systems at different temperatures to exchange complete configurations. Thus, the inclusion of higher temperature systems ensures that the lower temperature systems can access a representative set of low-temperature regions of phase space. This concept is illustrated in Figure 1.

Simulation of $M$ replicas, rather than one, requires on the order of $M$ times more computational effort. This 'extra expense' of parallel tempering is one of the reasons for the initially slow adoption of the method. Eventually, it became clear that a parallel tempering simulation is more than $1 / M$ times more efficient than a standard, single-temperature Monte Carlo simulation. This increased efficiency derives from allowing the lower temperature systems to sample regions of phase space that they would not have been able to access had regular sampling been conducted for a single-temperature simulation that was $M$ times as long. While not essential to the method, it is also the case that parallel tempering can make efficient use of large CPU clusters, where different replicas can be run in parallel. An additional benefit of the parallel tempering method is the generation of results for a range of temperatures, which may also be of interest to the investigator. It is now widely appreciated that parallel tempering is a useful and powerful computational method.

One of the debated issues in parallel tempering regards the details of the exchange, or swapping, of configurations between replicas. Pertinent questions include how many different replicas and at what temperatures to use, and how frequently swaps should be attempted, and the relative computational effort to expend on the different replicas. Another emerging issue is how to swap only part of the system, so as to overcome the growth as $\sqrt{N}$ of the number replicas required to simulate a system of size $N$. We address these points of controversy in this review.

The widespread use of parallel tempering in the simulation field has led to the emergence of a number of new issues. It has also become clear that temperature may not always be the best parameter to 
temper, and parallel tempering can be conducted with order parameters other than temperature, such as pair potentials or chemical potentials. Of interest is how to choose the order parameter whose swapping will give the most efficient equilibration. It has become clear that multi-dimensional parallel tempering is possible. That is, swapping between a number of parameters in the same simulation, in a multi-dimensional space of order parameters, is feasible and sometimes advised. The improvement in sampling resulting from the use of parallel tempering has revealed deficiencies in some of the most popular force fields used for atomistic simulations, and it would seem that the use of parallel tempering will be essential in tests of new and improved force fields.

Parallel tempering can be combined with most other simulation methods, as the exchanges, if done correctly, maintain the detailed balance or balance condition of the underlying simulation. Thus, there is almost an unlimited scope for the utilization of the method in computer simulation. This leads to intriguing possibilities, such as combining parallel tempering with quantum methods.

\section{Theory}

\subsection{Theory of Monte Carlo Parallel Tempering.}

In a typical parallel tempering simulation we have $M$ replicas, each in the canonical ensemble, and each at a different temperature, $T_{i}$. In general $T_{1}<T_{2}<\ldots<T_{M}$, and $T_{1}$ is normally the temperature of the system of interest. Since the replicas do not interact energetically, the partition function of this larger ensemble is given by

$$
Q=\prod_{i=1}^{M} \frac{q_{i}}{N !} \int d \mathbf{r}_{i}^{N} \exp \left[-\beta_{i} U\left(\mathbf{r}_{i}^{N}\right)\right]
$$

where $q_{i}=\prod_{j=1}^{N}\left(2 \pi m_{j} k_{\mathrm{B}} T_{i}\right)^{3 / 2}$ comes from integrating out the momenta, $m_{j}$ is the mass of atom $j$, $\mathbf{r}_{i}^{N}$ specifies the positions of the $N$ particles in system $i, \beta_{i}=1 /\left(k_{\mathrm{B}} T_{i}\right)$ is the reciprocal temperature, and $U$ is the potential energy, or the part of the Hamiltonian that does not involve the momenta. If the probability of performing a swap move is equal for all conditions, exchanges between ensembles $i$ and $j$ are accepted with the probability

$$
A=\min \left\{1, \exp \left[+\left(\beta_{i}-\beta_{j}\right)\left(U\left(\mathbf{r}_{i}^{N}\right)-U\left(\mathbf{r}_{j}^{N}\right)\right)\right]\right\} .
$$

Swaps are normally attempted between systems with adjacent temperatures, $j=i+1$.

Parallel tempering is an exact method in statistical mechanics, in that it satisfies the detailed balance or balance condition, ${ }^{7}$ depending on the implementation. This is an important advantage of parallel tempering over simulated annealing, as ensemble averages cannot be defined in the latter method. 
Parallel tempering is complementary to any set of Monte Carlo moves for a system at a single temperature, and such single-system moves are performed between each attempted swap. To satisfy detailed balance, the swap moves must be performed with a certain probability, although performing the swaps after a fixed number of single-temperature Monte Carlo moves satisfies the sufficient condition of balance. ${ }^{7}$ A typical sequence of swaps and single-temperature Monte Carlo moves is shown in Figure 2.

Kofke conducted an analysis of the average acceptance rate, $\langle A\rangle$, of exchange trials and argued that this quantity should be related to the entropy difference between phases. ${ }^{8-10}$ For systems assumed to have Gaussian energy distributions, typical of many systems that are studied using computer simulation, see Figure 3, he found the average acceptance ratio, $\langle A\rangle$, to be given by

$$
\langle A\rangle=\operatorname{erfc}\left[\left(\frac{1}{2} C_{v}\right)^{1 / 2} \frac{1-\beta_{j} / \beta_{i}}{\left(1+\left(\beta_{j} / \beta_{i}\right)^{2}\right)^{1 / 2}}\right],
$$

where $C_{v}$ is the heat capacity at constant volume, which is assumed to be constant in the temperature range between $\beta_{i}$ and $\beta_{j}$. Simply put, the acceptance rate for the trials depends on the likelihood that the system sampling the higher temperature happens to be in a region of phase space that is important at the lower temperature. This theoretical analysis of the acceptance rates becomes useful when considering the optimal choice of temperatures for a parallel tempering simulation (see Section 2.3).

\subsection{Theory of Molecular Dynamics Parallel Tempering.}

In Monte Carlo implementations of parallel tempering, we need only consider the positions of the particles in the simulation. In molecular dynamics, we must also take into account the momenta of all the particles in the system. Sugita and Okamoto proposed a parallel tempering molecular dynamics method in which after an exchange, the new momenta for replica $i, p^{(i)^{\prime}}$, should be determined as

$$
p^{(i)^{\prime}}=\sqrt{\frac{T_{\text {new }}}{T_{\text {old }}}} p^{(i)},
$$

where $p^{(i)}$ are the old momenta for replica $i$, and $T_{\text {old }}$ and $T_{\text {new }}$ are the temperatures of the replica before and after the swap, respectively. ${ }^{6}$ This procedure ensures the average kinetic energy remains equal to $\frac{3}{2} N k_{\mathrm{B}} T$. The acceptance criterion for an exchange remains the same as for the MC implementation (Eqn. 2) and satisfies detailed balance.

When doing parallel tempering molecular dynamics, one must take care in the interpretation of the results. A parallel tempering exchange is an 'unphysical' move, and so one cannot draw conclusions about dynamics. That is, when using parallel tempering molecular dynamics, one is only really doing a form of sampling and not 'true' molecular dynamics. 


\subsection{Optimal Choice of Temperatures.}

How one chooses both the number of replicas employed in a parallel tempering simulation and the temperatures of the replicas are questions of great importance. One wishes to achieve the best possible sampling with the minimum amount of computational effort. The highest temperature must be sufficiently high so as to ensure that no replicas become trapped in local energy minima, while the number of replicas used must be large enough to ensure swapping occurs between all adjacent replicas. Several suggestions for the number and temperature of the replicas have been offered. It is clear from Figure 3 and Eqn. 22 that the energy histograms must overlap for swaps to be accepted. Sugita $e t$ $a l$. and Kofke have proposed that the acceptance probability could be made uniform across all of the different replicas, in an attempt to ensure that each replica spends the same amount of simulation time at each temperature. ${ }^{6,8,9}$ Kofke showed that a geometric progression of temperatures ( $\frac{T_{i}}{T_{j}}=$ constant) for systems in which $C_{v}$ is constant across the temperatures results in equal acceptance ratios. Sanbonmatsu $e t a l$. suggested that a target acceptance ratio, $A_{\text {target }}$, can be obtained iteratively by solving

$$
A_{\text {target }}=\exp [\Delta \beta \Delta E],
$$

where $\Delta E$ is the difference in the average energies of the systems in adjacent temperatures. ${ }^{11}$ Iterative methods for adjusting the temperatures of the different systems to ensure that acceptance ratios stay within acceptable bounds had previously been proposed and utilized by Falcioni ${ }^{12}$ and Schug $e t$ $a l .{ }^{13}$ in adaptive temperature control schemes. Rathore et al. ${ }^{14}$ extended these approaches to suggest a scheme for the optimal allocation of temperatures to replicas that is also based on iteratively altering system temperatures. In their scheme, the lowest temperature is fixed, and the other system temperatures are determined by iteratively solving

$$
\left.\frac{\Delta E}{\sigma_{m}}\right|_{T_{j}}=\left[\frac{\Delta E}{\sigma}\right]_{\text {target }},
$$

for each of the temperatures, $T_{j}$, where $\sigma_{m}=\left[\sigma\left(T_{j}\right)+\sigma\left(T_{i}\right)\right] / 2$ is the average deviation of the energies in the two systems. One can choose the target value to achieve a desired acceptance ratio.

Rathore et al. also consider the optimal acceptance ratio and number of replicas in parallel tempering simulations. ${ }^{14}$ For the case studies used in their work, they found that an acceptance ratio of $20 \%$ yielded the best possible performance. That is, adding more replicas once the high and low temperatures are fixed and the acceptance ratio of $20 \%$ had been achieved resulted in no increase in the performance of the simulation.

Recently, Kone and Kofke have provided an analysis of the selection of temperature intervals in systems where $C_{v}$ is assumed to be piecewise constant across each temperature interval. ${ }^{15}$ They argue that although this may not always be the case, the assumption is reasonable and does not require an iterative scheme that can consume valuable CPU time and which violates detailed balance. Their analysis is based on maximising the mean square displacement, $\sigma^{2}$, of a system as it performs the 
random walk over temperatures. The value of $\sigma^{2}$ is proportional to the number of accepted swaps and $\left(\ln \left(\beta_{j} / \beta_{i}\right)\right)^{2}$. By maximizing $\sigma^{2}$ with respect to the acceptance probability, they found that an acceptance probability of $23 \%$ is optimal. This value is strikingly similar to the empirically determined $20 \%$ of Rathore et al. Kone and Kofke suggest "tuning" the temperature intervals to achieve the $23 \%$ acceptance probability during the initial equilibration of a simulation. This approach appears to be an efficient method to select temperature intervals in parallel tempering simulations that mix efficiently.

A similar scheme for choosing the temperatures has recently been proposed by Katzgraber et al., which uses an adaptive feedback-optimized algorithm to minimize round-trip times between the lowest and highest temperatures. ${ }^{16}$ This approach more directly characterizes the mixing between the high and low temperature systems. In complex cases, where there are subtle bottlenecks in the probability of exchange of configurations, the round-trip time is likely to better characterize the overall efficiency of parallel tempering than is the average acceptance probability. The approach of Katzgraber et al. is a promising one for such complex cases.

A related issue is how much simulation effort should be expended on each replica. For example, it would seem that the low temperature replicas would benefit from additional simulation effort, as the correlation times at lower temperature are longer. This issue is untouched in the literature.

Since the width of the energy histograms increases as $\sqrt{N}$, but the average energy increases as $N$, the number of replicas increases as $\sqrt{N}$, where $N$ is is the system size. ${ }^{5}$ One, therefore, would like a method where only part of the configurational degrees of freedom are exchanged. Interestingly, this issue was solved in Swendsen and Wang's 1986 paper for spin systems, ${ }^{1}$ but it has not been solved in an exact, efficient way for atomistic systems. The main difficulty seems to be in defining a piece of a system that can be exchanged without the penalty of a large surface energy.

\subsection{Parallel Tempering with Alternative Parameters and Sampling Methods.}

The general idea of parallel tempering is not limited to exchanges or swaps between systems at different temperatures. Investigators have developed a number of methods based on swapping alternative parameters in order to minimize barriers that inhibit correct sampling. Additionally, parallel tempering can be combined with a large number of alternative sampling methods, and its use has led to a great improvement in the sampling of many existing computational methods.

Fukunishi et al. developed a Hamiltonian parallel tempering method that they applied to biomolecular systems. ${ }^{17}$ In this approach, only part of the interaction energy between particles is scaled between the different replicas. In their work, they conduct case studies using two different implementations of their approach. In the first they scale hydrophobic interactions between replicas. In the second, they scale the van der Waals interactions between replicas by introducing a cut-off in the interaction, effectively allowing chains of atoms to pass through each other. The acceptance probability in Hamiltonian parallel tempering for a swap between replicas $i$ and $j$ is given by

$$
A=\min \left\{1, \exp \left[-\beta\left(\left[H_{i}\left(X^{\prime}\right)+H_{j}(X)\right]-\left[H_{i}(X)+H_{j}\left(X^{\prime}\right)\right]\right)\right]\right\},
$$


where $H_{i}(X)$ is the Hamiltonian of configuration $X$ in replica $i$, and configurations $X$ and $X^{\prime}$ are the configurations in replicas $i$ and $j$, respectively, prior to the swap attempt.

Parallel tempering using multiple swapping variables was first proposed and developed by Yan and de Pablo. ${ }^{18,19}$ Instead of considering a one-dimensional array of replicas at different temperatures, they suggested using an $n$-dimensional array, where each dimension represented a parameter that varied between replicas. Swaps both within and between dimensions were allowed in their scheme. In their first work they conducted parallel tempering between different temperatures and chemical potentials in the grand canonical ensemble, but the scheme they proposed was general. They showed that extensions of parallel tempering to multiple dimensions are limited only by the imagination of the investigator in choosing the variables to swap and the available computational resources. Sugita et al. utilized multdimensional exchanges in molecular dynamics studies. ${ }^{20}$

de Pablo and co-workers also implemented parallel tempering in the multicanonical ensemble. ${ }^{21}$ In the multicanonical ensemble, the probability distribution is no longer Boltzmann, but becomes

$$
p\left(\mathbf{r}^{N}\right)=(\text { const }) e^{-\beta U\left(\mathbf{r}^{N}\right)} w\left(\mathbf{r}^{N}\right)
$$

The weight factors, $w\left(\mathbf{r}^{N}\right)$, are chosen so as to lower the barriers in the system. de Pablo and coworkers derived multicanonical weights by an iterative process using a Boltzmann inversion of histograms. Another way to write Eq. (8) is to use instead of the Hamiltonian $U$, the weighted Hamiltonian $U+\xi(U)$ when attempting swap moves, where $\xi(U)$ is an umbrella potential. By using a multicanonical ensemble, de Pablo and co-workers were able to reduce the number of replicas required in their simulation, due to a broader overlap of thermodynamic-property histograms. In general, when combined with a multicanonical simulation, a short parallel tempering run can be performed, and the multicanonical weight factors can be determined by using histogram reweighting. These weights can then be used in the multicanonical part of the calculation. ${ }^{22}$ Parallel tempering can be combined with a multicanonical simulation. That is, in the multicanonical simulation, a number of replicas, each in the multicanonical ensemble but each with different multicanonical weight factors covering different energy ranges, may be employed..$^{22}$ It should be noted that far fewer replicas are needed in this method than in typical parallel tempering because the energy ranges covered in a multicanonical simulation are far wider than in a canonical simulation. The weight factors utilized in these methods may then be iteratively improved during the equilibration period as the simulation proceeds, using histogram reweighting techniques.

In free energy perturbation calculations, a parameter $\lambda$ is introduced. One wishes to compute the free energy difference to go from an initial $(\lambda=0)$ state and a final $(\lambda=1)$ state. For parallel tempering with free energy perturbation one can consider $M$ replicas, each with a different $\lambda$ parameter, where each replica has a slightly different Hamiltonian

$$
U_{\lambda}=U_{\lambda=0}+\lambda\left(U_{\lambda=1}-U_{\lambda=0}\right) .
$$


Swaps may be attempted between replicas using the Hamiltonian acceptance criterion (Eq. 7), and the free energy difference between two lambda parameters can be determined as in regular free energy calculations. Of course, one may utilize a number of different temperature replicas for each value of $\lambda$ in a multidimensional approach. Use of parallel tempering in multicanonical simulations, free energy calculations, and umbrella sampling is growing. ${ }^{20-26}$

One of the most fruitful combinations of parallel tempering with existing sampling techniques has been with density of states methods based on Wang-Landau sampling. ${ }^{27}$ Density of states methods are similar to multicanonical ones in that the weight factor is the reciprocal of the density of states. However, in density of states methods a random walk in energy space is conducted, and a running estimate of the inverse of the density of states as a function of the energy is performed. Alternatively the configurational temperature is collected as a function of the energy and the density of states determined by integrating the inverse temperature. ${ }^{28}$ Other sets of conjugate variables can also profitably be used. ${ }^{29}$ These methods effectively circumvent the tedious and time consuming process of calculating weight factors in multicanonical simulations. de Pablo and co-workers have proposed extended ensemble density of states methods where overlapping windows or replicas of different energy or reaction/transition coordinate values are utilized. ${ }^{30}$ Configurational swaps between windows are attempted at regular intervals to prevent the simulations in the parallel replicas from becoming stuck in non-representative regions of phase space. A combination of density of states methods and parallel tempering has successfully been used to study protein folding ${ }^{31-33}$ and solid-liquid equilibria. ${ }^{34}$

Vlugt and Smit applied parallel tempering to the transition path sampling method. ${ }^{35}$ They showed that parallel tempering conducted between different temperatures and between different regions along transition paths is able to overcome the problem of multiple saddle points on a free energy surface. Parallel tempering transition path sampling can provide for more accurate estimates of transition rates between stable states than single-temperature Monte Carlo transition path sampling.

Parallel tempering has been combined with a number of other computational methods, and in almost all cases its use has resulted in better sampling and an increase in the accuracy of the computational method. Prominent examples include parallel tempering with cavity bias to study the phase diagram of Lennard-Jones fluids, ${ }^{36}$ with analytical rebridging for the simulation of cyclic peptides, ${ }^{37}$ and with the wormhole algorithm to explore the phase behavior of random copolymer melts. ${ }^{38}$

Very recently an extension to parallel tempering, known as Virtual-Move Parallel Tempering, has been proposed by Coluzza and Frenkel. ${ }^{39}$ In their scheme they include information about all possible parallel tempering moves between all replicas in the system, rather than just between adjacent replicas, when accumulating statistical averages. This approach is essentially a parallel tempering version of the "waste recyling" Monte Carlo method of Frenkel ${ }^{40}$ and has been shown to improve statistical averaging by upto a factor of 20 . 


\subsection{Non-Boltzmann Distributions.}

Since their introduction in the late 1980s, Tsallis statistics have become increasingly important in statistical mechanics. ${ }^{41}$ Due to their power-law, rather than Boltzmann, properties, Tsallis statistics generally lead to smaller energy barriers. Therefore, optimization with Tsallis, rather than Boltzmann, statistics can be very useful in energy minimization problems. Whitfield et al. have developed a version of the parallel tempering algorithm that is based upon Tsallis statistics. ${ }^{42}$ This method has been used, for example, for fast conformational searches of peptide molecules. ${ }^{43}$

\section{Applications}

\subsection{Polymers.}

Simulations of polymeric systems are notoriously difficult due to chain tangling, the high density of the systems of interest, and the large system sizes required to accurately model high molecular weight species. The first application of parallel tempering to polymeric systems was by Yan and de Pablo to high molecular weight species. ${ }^{19}$ Bunker and Dunweg ${ }^{44}$ were the first to utilize excluded volume parallel tempering, where different replicas have different core potentials. They studied polymer melts for polymer chain lengths ranging from 60 to 200 monomers. Their method created a thermodynamic path from the full excluded volume system to an ideal gas of random walks and increased the efficiency of all their simulations. Bedrov and Smith ${ }^{45}$ studied fully atomistic polymer melts of 1,4-polybutadiene at a range of temperatures, performing parallel tempering swaps isobarically. They showed that their parallel tempering approach provided a substantial improvement in equilibration and sampling of conformational phase space when compared to regular MD simulations. See Figure 4. Theodorou and co-workers studied cis-1,4 polyisoprene melts using parallel tempering and once again found that use of parallel tempering resulted in far quicker equilibration over a range of temperatures. ${ }^{46}$ More recently, Banaszak et al. have utilized hyperparallel tempering in an osmotic ensemble to study the solubility of ethylene in low-density polyethylene. ${ }^{47}$ Using their novel method they were able to examine the effect of both polyethylene chain length and branching on the solubility of ethylene.

\subsection{Proteins.}

Biological systems, particularly proteins, are computationally challenging because they have particularly rugged energy landscapes that are difficult for regular Monte Carlo and molecular dynamics techniques to traverse. Hansmann was the first to apply parallel tempering to biological molecules in a Monte Carlo based study of the simple 7-amino acid Met-enkephalin peptide. ${ }^{4}$ Hansmann showed that parallel tempering based simulations could overcome the "simulation slowdown" problem and were more efficient than regular canonical Monte Carlo simulations. The application of parallel tempering to biological problems, however, did not take-off until Sugita and Okamoto's work that introduced the use of molecular dynamics parallel tempering. ${ }^{6}$ They applied their approach to Met-enkephalin and 
demonstrated that their parallel tempering based method did not get trapped in local energy minima, unlike regular microcanonical molecular dynamics simulations of the same molecule.

Following demonstration of the power of parallel tempering for molecular systems, its use in the biological simulation community rapidly expanded. Parallel tempering has been used to determine folding free energy contour maps for a number of proteins, revealing details about folding mechanisms and intermediate state structure ${ }^{48-52}$ and has facilitated the simulation of membrane proteins. ${ }^{53-56}$ Parallel tempering has proved to be particularly powerful when applied to NMR structure refinement and in the interpretation of data from NMR, ${ }^{57-61}$ circular dichroism, ${ }^{62}$ IR spectra, ${ }^{63}$ and electric deflection data ${ }^{64}$ of proteins and peptides. For models of globular proteins and oligomeric peptides, parallel tempering has been used to study previously unexplored regions of phase diagrams and to sample aggregate transitions. ${ }^{65,66}$ In the study of sucrose solutions near the glass transition temperature, parallel tempering simulations showed a much better fit to experimental data than did conventional NPT MC results. ${ }^{67}$ Other interesting work using parallel tempering includes studies of the thermodynamics of fibril formation using an intermediate resolution protein model ${ }^{68}$ and of the hypervariable regions of an antibody domain where the chief interactions governing conformational equilibria in these systems were determined. ${ }^{6}$

With this increased sampling ability of parallel tempering has come the realization that current force fields for biological simulation are lacking in some respects. Parallel tempering simulations of solvated biological molecules have also revealed deficiencies in popular implicit solvent models. ${ }^{52,70,71}$ As parallel tempering can also be used with explicit solvent models, the differences between the treatments can be determined, and in the future such simulations could be used to improve implicit solvent models.

Brooks and co-workers have developed a multiscale modeling toolkit that can interface with the popular CHARMM and AMBER molecular simulation codes. ${ }^{72}$ Parallel tempering is implemented in the toolkit to allow enhanced sampling and is used to study the ab initio folding of peptides from first principles. Parallel tempering has clearly become the method of choice in ab initio protein folding as evidenced by the the work of Skolnick and co-workers, ${ }^{73}$ Garcia and Sanbonmatsu, ${ }^{11,74,75}$ and Yang et al. $^{76}$

When examining the biological and chemical literature of parallel tempering, it is apparent that the vast majority of work is based on molecular dynamics, rather than Monte Carlo. As one is not doing 'true' MD when using parallel tempering, there is no reason why Monte Carlo methodologies cannot be implemented more frequently in the biological and chemical communities. Indeed, we expect this to be a promising avenue for future research.

\subsection{Solid state.}

Crystal structure solution provided one of the first mainstream atomistic simulation examples of the 
power of parallel tempering. Falcioni and Deem used parallel tempering in a biased MC scheme to determine the structures of zeolites from powder diffraction data. ${ }^{77}$ For complicated zeolite structures containing more than eight unique tetrahedral atoms, simulated annealing is unable to solve the crystal structures. However, parallel tempering simulations were shown to be able to solve the structures of all complex zeolites, including the most complicated zeolite structure, ZSM-5, which contains twelve unique tetrahedral atoms. ZefsaII has since been successfully used to solve the structures of at least a dozen newly synthesized zeolites and is freely downloadable on the web. A similar approach to crystal structure determination from powder diffraction data has been implemented by Favre-Nicolin et al. ${ }^{78}$ and this method has been successful in solving several structures. ${ }^{79-81}$

A seminal simulation study of the rate of crystal nucleation by Auer and Frenkel utilized the parallel tempering method by allowing swaps between 'windows' at different points along the reaction coordinate from the liquid to solid state. ${ }^{82}$ This work introduced, for the first time, the ability to calculate nucleation rates from first principles.

Other examples of solid-state parallel tempering simulations include the computation of sodium ion distributions in zeolites, ${ }^{83}$ studying the finite temperature behavior of $\mathrm{C}_{60}$ clusters, ${ }^{84}$ the simulation of $\mathrm{Si}$ surfaces, ${ }^{85,86}$ and the explanation of the titration behavior of $\mathrm{MbCO}$ over a range of $\mathrm{pH}$ values. ${ }^{87}$

\subsection{Spin glass.}

Spin glasses have provided a severe test of the effectiveness of parallel tempering. ${ }^{88}$ In the Parisi solution of the infinite range Edwards-Anderson model, widely believed by many but not all physicists to apply to finite-range spin glasses as well, there is a finite energy for excitations above the ground state, and the boundary of these excitations has a space-filling structure. Initial simulations for the Edwards-Anderson model confirmed the finite excitation energy. ${ }^{89}$ Initial suggestions for a fractal surface ${ }^{89}$ were ruled out by later simulations. ${ }^{90}$ For the vector spin glass model, the excitation energy was again found to be finite. ${ }^{91}$ Initial suggestions of a fractal surface were also largely ruled out in later simulations. ${ }^{92}$

\subsection{Quantum.}

Quantum level systems, whilst being far more computationally demanding than classical systems, may benefit from the improved sampling provided by parallel tempering. So far, the main application of parallel tempering at the quantum level has been in studies of phase transitions and in the location of energy minima in complex systems. Parallel tempering is ideal for these studies, as dynamics are not of interest. Okamoto and co-workers conducted parallel tempering based ab initio correlated electronic structure calculations. ${ }^{93}$ In their studies of Li clusters, they demonstrated that parallel tempering could be successfully applied to systems described with a high level of detail. Sengupta et al. combined quantum Monte Carlo with parallel tempering to study the phase diagram of a 1-D Hubbard model. ${ }^{94}$ Quantum parallel tempering was found to significantly reduce "sticking" effects, where the simulation gets stuck in the incorrect phase close to the phase boundary. 
Shin et al. have studied quantum phase transitions of water clusters, ${ }^{95}$ where the rotational modes can be highly quantum. Parallel tempering allowed for efficient conformational sampling. They remark that "combining Car-Parrinello approach with replica exchange [parallel tempering] and path integral molecular dynamics can provide an ideal methodology for studying quantum behavior of clusters." Although the suggested approach is highly computationally expensive, it may become increasingly feasible in future years. Parallel tempering has also been successfully employed in a study of the finite temperature optical spectroscopy of $\mathrm{CaAr}_{n}$ clusters $^{96}$ and in quantum path integral simulations of the solid-liquid phase diagrams of $\mathrm{Ne}_{13}$ and (para- $\left.\mathrm{H}_{2}\right)_{13}$ clusters. ${ }^{97}$

\subsection{General Optimization Problems.}

Parallel tempering has been successfully used in a number of general optimization problems. Habeck et al. developed a sampling algorithm for the exploration of probability densities that arise in Bayesian data analysis. ${ }^{98}$ Their approach utilized Tsallis statistics, and the effectiveness of parallel tempering was demonstrated by interpreting experimental NMR data for a folded protein. In image analysis, parallel tempering has been shown to lead to an improvement by a factor of two for both success rate and mean position error when compared to simulated annealing approaches. ${ }^{99}$ Parallel tempering has also been utilized to locate the global minima of complex and rugged potential energy surfaces that arise in atomistic models of receptor-ligand docking ${ }^{100}$ and in risk analysis. ${ }^{101}$

\section{Conclusion}

In this review we have given an overview of the history of parallel tempering. We have described the basic theory and many of the extensions to the original method. Several examples in a variety of physiochemical arenas have been discussed. Highlighted technical aspects to sort out include best allocations to cluster computers, ${ }^{102}$ determination of the optimal amount of simulation effort to expend on each replica, and partial swapping of partial configuration information for atomistic systems.

A number of potential new areas for application of parallel tempering occur to us. One rather large one is the application of parallel tempering, rather than simulated annealing, ${ }^{103}$ to X-ray single-crystal structure solution. A related issue is the prediction of polymorphs for crystals of small, organic drug molecules. Also related is use of parallel tempering in rational drug design-most current approaches use grid searching, traditional Monte Carlo, or at best simulated annealing. ${ }^{104}$ Another physical application where enhanced sampling might be of use is in field theories for polymeric systems with non-

trivial phase structure. ${ }^{105}$ Also possible would be the complementary inclusion in $a b$ initio molecular dynamics, if sampling only is desired. Even experimental applications could be possible in materials discovery ${ }^{106}$ or laboratory protein evolution. ${ }^{107}$ 


\section{References}

[1] R. H. Swendsen and J.-S. Wang, Phys. Rev. Lett., 1986, 57, 2607.

[2] C. J. Geyer In Computing Science and Statistics: Proceedings of the 23rd Symposium on the Interface, p. 156, New York, 1991. American Statistical Association.

[3] C. J. Geyer and E. A. Thompson, J. Am. Stat. Assn., 1995, 90, 909-920.

[4] U. H. E. Hansmann, Chem. Phys. Lett., 1997, 281, 140.

[5] M. Falcioni and M. W. Deem, J. Chem. Phys., 1999, 110, 1754.

[6] Y. Sugita and Y. Okamoto, Chem. Phys. Lett., 1999, 314, 141.

[7] V. I. Manousiouthakis and M. W. Deem, J. Chem. Phys., 1999, 110, 2753.

[8] D. A. Kofke, J. Chem. Phys., 2002, 117, 6911.

[9] D. A. Kofke, J. Chem. Phys., 2004, 120, 10852.

[10] D. A. Kofke, J. Chem. Phys., 2004, 121, 1167.

[11] K. Y. Sanbonmatsu and A. E. Garcia, Proteins, 2002, 46, 225.

[12] M. Falcioni, unpublished.

[13] A. Schug, Proteins, 2004, 57, 792.

[14] N. Rathore, M. Chopra, and J. J. de Pablo, J. Chem. Phys., 2005, 122, 024111.

[15] A. Kone and D. A. Kofke, J. Chem. Phys., 2005, 122, 206101.

[16] D. Huse, H. G. Katzgraber, S. Trebst, and M. Troyer In Europhysics Conference on Computational Physics 2004, Book of Abstracts, p. 63. European Physical Society, 2004.

[17] H. Fukunishi, O. Watanabe, and S. Takada, J. Chem. Phys., 2002, 116, 9058.

[18] Q. Yan and J. J. de Pablo, J. Chem. Phys., 1999, 111, 9509.

[19] Q. Yan and J. J. de Pablo, J. Chem. Phys., 2000, 113, 1276.

[20] Y. Sujita, A. Kitao, and Y. Okamoto, J. Chem. Phys., 2000, 113, 6042.

[21] R. Faller, Q. Yan, and J. J. de Pablo, J. Chem. Phys., 2002, 116, 5419. 
[22] Y. Sugita and Y. Okamoto, Chem. Phys. Lett., 2000, 329, 261.

[23] A. Mitsutake, Y. Sugita, and Y. Okamoto, J. Chem. Phys., 2003, 118, 6664.

[24] A. Mitsutake, Y. Sugita, and Y. Okamoto, J. Chem. Phys., 2003, 118, 6676.

[25] K. Murata, Y. Sugita, and Y. Okamoto, Chem. Phys. Lett., 2004, 385, 1.

[26] Y. Okamoto, J. Molec. Graph. Mod., 2004, 22, 425.

[27] F. Wang and D. Landau, Phys. Rev. Lett., 2001, 86, 2050.

[28] Q. Yan and J. J. de Pablo, Phys. Rev. Lett., 2003, 90, 035701.

[29] M. Fasnacht, R. H. Swendsen, and J. M. Rosenberg, Phys. Rev. E, 2004, 69, 056704.

[30] N. Rathore and J. J. de Pablo, J. Chem. Phys., 2002, 117, 7781.

[31] N. Rathore, T. A. Knotts, and J. J. de Pablo, J. Chem. Phys., 2003, 118, 4285.

[32] N. Rathore, T. A. Knotts, and J. J. de Pablo, Biophysical J., 2003, 85, 3963.

[33] N. Rathore, Q. Yan, and J. J. de Pablo, J. Chem. Phys., 2004, 120, 5781.

[34] E. A. Mastny and J. J. de Pablo, J. Chem. Phys., 2005, 122, 124109.

[35] T. J. H. Vlugt and B. Smit, Chem. Phys. Comm., 2001, 2, 1.

[36] V. Ortiz, J. R. Maury-Evertsz, and G. E. Lopez, Chem. Phys. Lett., 2003, 368, 452.

[37] M. G. Wu and M. W. Deem, J. Chem. Phys., 1999, 111, 6625.

[38] J. Houdayer and M. Muller, Macromolecules, 2004, 37, 4283.

[39] I. Coluzza and D. Frenkel, submitted.

[40] D. Frenkel, Proc. Natl. Acad. Sci. USA, 2004, 101, 17571.

[41] C. Tsallis, J. Stat. Phys., 1988, 52, 479.

[42] T. M. Whitfield, L. Bu, and J. E. Straub, Physica A, 2002, 305, 157.

[43] S. Jang, S. Shin, and Y. Pak, Phys. Rev. Lett., 2003, 91, 058305.

[44] A. Bunker and B. Dunweg, Phys. Rev. E, 2000, 63, 016701.

[45] D. Bedrov and G. D. Smith, J. Chem. Phys., 2001, 115, 1121. 
[46] M. Dozastakis, V. G. Mavrantzas, and D. N. Theodorou, J. Chem. Phys., 2001, 115, 11352.

[47] B. J. Banaszak, R. Faller, and J. J. de Pablo, J. Chem. Phys., 2004, 120, 11304.

[48] R. Zhou, J. Molec. Graph. Mod., 2004, 22, 451.

[49] R. Zhou and B. J. Berne, Proc. Natl. Acad. Sci. USA, 2002, 99, 12777.

[50] R. Zhou, Proc. Natl. Acad. Sci. USA, 2003, 100, 13280.

[51] A. E. Garcia and J. E. Onuchic, Proc. Natl. Acad. Sci. USA, 2003, 100, 13898.

[52] R. Zhou, B. J. Berne, and R. Germain, Proc. Natl. Acad. Sci. USA, 2001, 98, 14931.

[53] W. Im and C. L. B. III, J. Mol. Bio., 2004, 337, 513.

[54] W. Im, M. Feig, and C. L. B. III, Biophysical J., 2003, 85, 2900.

[55] H. Kokubo and Y. Okamoto, J. Chem. Phys., 2004, 120, 10837.

[56] H. Kokubo and Y. Okamoto, Chem. Phys. Lett., 2004, 392, 168.

[57] T. Haliloglu, A. Kolinski, and J. Skolnick, Biopolymers, 2003, 70, 548.

[58] G. L. Penna, A. Mitsutake, M. Masayu, and Y. Okamoto, Chem. Phys. Lett., 2003, 380, 609.

[59] J. Chen, H.-S. Won, W. Im, H. J. Dyson, and C. L. B. III, J. Biomolec. NMR, 2005, 31, 59.

[60] J. Chen, W. Im, and C. L. B. III, J. Am. Chem. Soc., 2004, 126, 16038.

[61] J. Vreede, W. Crielaard, K. J. Hellingwerf, and P. G. Bolhuis, Biophysical J., 2005, 88, 3525.

[62] G. S. Jas and K. Kuczera, Biophysical J., 2004, 87, 3786.

[63] S. Gnanakaran, R. M. Hochstrasser, and A. E. Garcia, Proc. Natl. Acad. Sci. USA, 2004, 101, 9229.

[64] P. Dugourd, R. Antoine, G. Breaux, M. Broyer, and M. F. Jarrold, J. Am. Chem. Soc., 2005, 127, 4675 .

[65] D. L. Pagan, M. E. Grachava, and J. D. Gunton, J. Chem. Phys., 2004, 120, 8292.

[66] M. Cecchini, F. Rao, M. Seeber, and A. Caflisch, J. Chem. Phys., 2004, 121, 10748.

[67] N. C. Ekdawi-Sever, P. B. Conrad, and J. J. de Pablo, J. Phys. Chem. A, 2001, 105, 734. 
[68] H. D. Nguyen and C. K. Hall, Biophysical J., 2004, 87, 4122.

[69] M. K. Fenwick and F. A. Escobedo, Biopolymers, 2002, 68, 160.

[70] H. Nymeyer and A. E. Garcia, Proc. Natl. Acad. Sci. USA, 2003, 100, 13934.

[71] R. Zhou, Proteins, 2003, 53, 148.

[72] M. Feig, J. Karanicolas, and C. L. B. III, J. Molec. Graph. Mod., 2004, 22, 377.

[73] D. Kihara, H. Lu, A. Kolinski, and J. Skolnick, Proc. Natl. Acad. Sci. USA, 2001, 98, 10125.

[74] A. E. Garcia and K. Y. Sanbonmatsu, Proteins, 2001, 42, 345.

[75] K. Y. Sanbonmatsu and A. E. Garcia, Proc. Natl. Acad. Sci. USA, 2002, 99, 2782.

[76] W. Y. Yang, J. W. Pitera, W. C. Swope, and M. Gruebele, J. Mol. Bio., 2004, 336, 241.

[77] M. Falcioni and M. W. Deem, J. Chem. Phys., 1999, 110, 1754.

[78] V. Favre-Nicolin and R. Cerny, J. Appl. Cryst., 2002, 35, 734.

[79] M. C. Garcia-Cuesta, A. M. Lozano, J. J. Melendez-Martinez, F. Luna-Giles, A. L. Ortiz, L. M. Gonzalez-Mendez, and F. L. Cumbera, J. Appl. Cryst., 2004, 37, 993.

[80] P. Y. Zavalij, S. Yang, and M. S. Whittingham, Acta Cryst., 2003, B59, 753.

[81] E. Dova, R. Peschar, M. Sakata, K. Kato, A. F. Stassen, H. Schenk, and J. G. Haasnoot, Acta Cryst., 2004, B60, 528.

[82] S. Auer and D. Frenkel, Nature, 2001, 409, 1020.

[83] C. Beauvais, X. Guerrault, F.-X. Coudert, A. Boutin, and A. H. Fuchs, J. Phys. Chem. B, 2004, 108, 399.

[84] F. Calvo, J. Phys. Chem. B, 2001, 105, 2183.

[85] C. V. Ciobanu and C. Predescu, Phys. Rev. B, 2004, 70, 085321.

[86] F. C. Chuang, C. V. Ciobanu, C. Predescu, C. Z. Wang, and K. M. Ho, Surf. Sci., 2005, 578, 183.

[87] B. Rabenstein and E.-W. Knapp, Biophysical J., 2001, 80, 1141.

[88] E. Marinari, G. Parisi, and J. Ruiz-Lorenzo in Spin Glasses and Random Fields, ed. A. Young, Vol. 12 of Directions in Condensed Matter Physics; World Scientific, Singapore, 1998. 
[89] H. G. Katzgraber, M. Palassini, and A. P. Young, Phys. Rev. B, 2001, 63, 184422.

[90] H. G. Katzgraber and A. P. Young, Phys. Rev. B, 2002, 65, 214402.

[91] H. G. Katzgraber and A. P. Young, Phys. Rev. B, 2002, 65, 214401.

[92] H. G. Katzgraber, Comp. Phys. Comm., 2002, 147, 439.

[93] Y. Ishikawa, Y. Sujita, T. Nishikawa, and Y. Okamoto, Chem. Phys. Lett., 2001, 333, 199.

[94] P. Sengupta, A. W. Sandvik, and D. K. Campbell, Phys. Rev. B, 2002, 65, 155113.

[95] S. Shin, W. Son, and S. Jang, Theochem, 2004, 673, 109.

[96] F. Calco, F. Spiegelman, M. A. Gaveau, M. Briant, P. R. Fournier, J. M. Mestdagh, and J. P. Visticot, Euro. Phys. J. D, 2003, 24, 215.

[97] G. E. Lopez, J. Chem. Phys., 2002, 117, 2225.

[98] M. Habeck, M. Nilges, and W. Rieping, Phys. Rev. Lett., 2005, 94, 018105.

[99] C. Bertrand, M. Ohmi, R. Suzuki, and H. Kado, IEEE Trans. Biomed. Eng., 2001, $48,533$.

[100] H. Merlitz and W. Wenzel, Chem. Phys. Lett., 2002, 362, 271.

[101] H. Kozumi, Comp. Stat. Data Anal., 2004, 46, 441.

[102] D. J. Earl and M. W. Deem, J. Phys. Chem. B, 2004, 108, 6844.

[103] A. T. Brunger, J. Kuriyan, and M. Karplus, Science, 1987, 235, 458.

[104] R. S. DeWitte and E. I. Shakhnovich, J. Am. Chem. Soc., 1996, 118, 11733.

[105] A. Alexander-Katz, A. G. Moreira, S. W. Sides, and G. H. Fredrickson, J. Chem. Phys., 2005, 122, 014904.

[106] M. Falcioni and M. W. Deem, Phys. Rev. E, 2000, 61, 5948.

[107] L. D. Bogarad and M. W. Deem, Proc. Natl. Acad. Sci. USA, 1999, 96, 2591. 


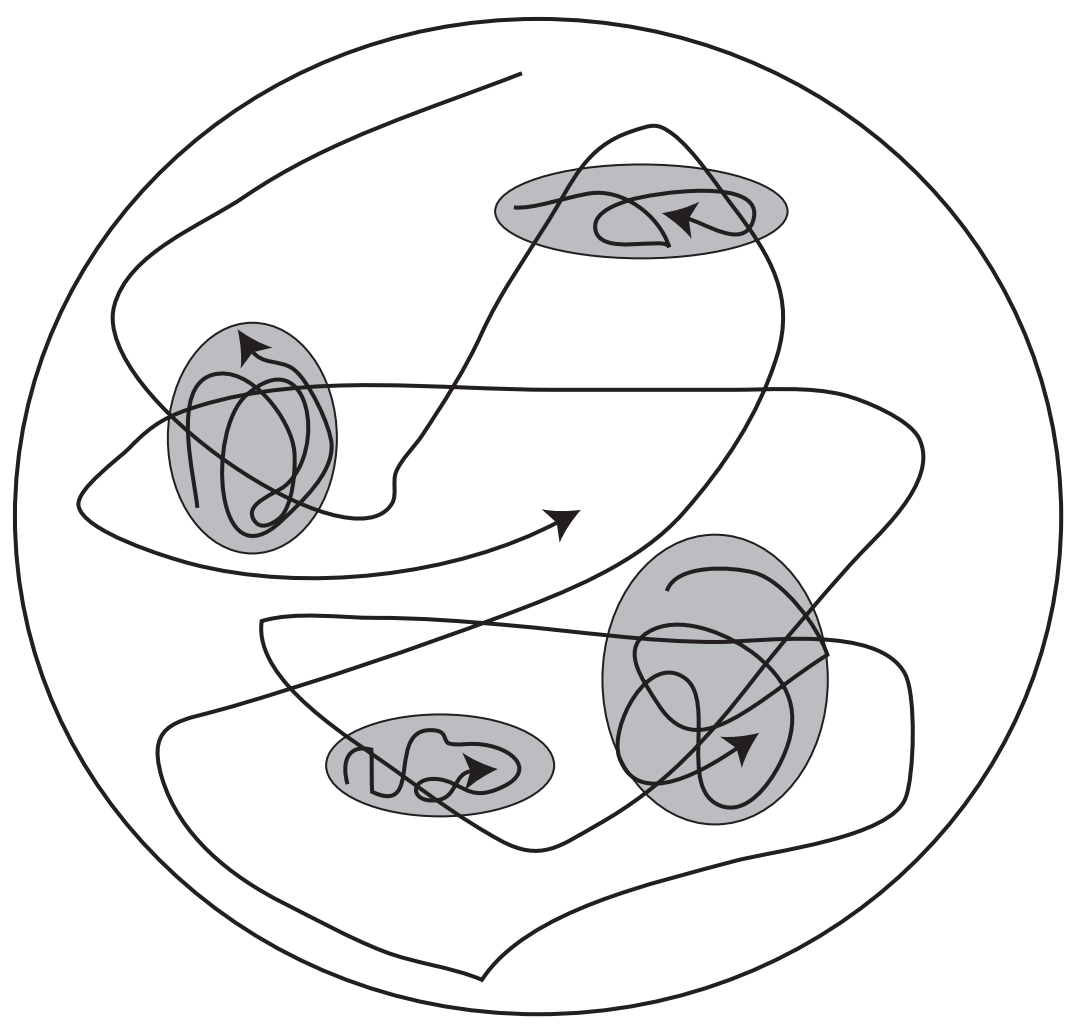

Figure 1: 2-D representation of phase space. A simulation at lower temperatures can become trapped in a non-representative sample of the low free energy minima (shaded regions). At higher temperatures, a simulation can sample more of phase space (light plus shaded areas). Configuration swaps between the lower and higher temperature systems allow the lower temperature systems to escape from one region of phase space where they were effectively 'stuck' and to sample a representative set of the low free energy minima. 


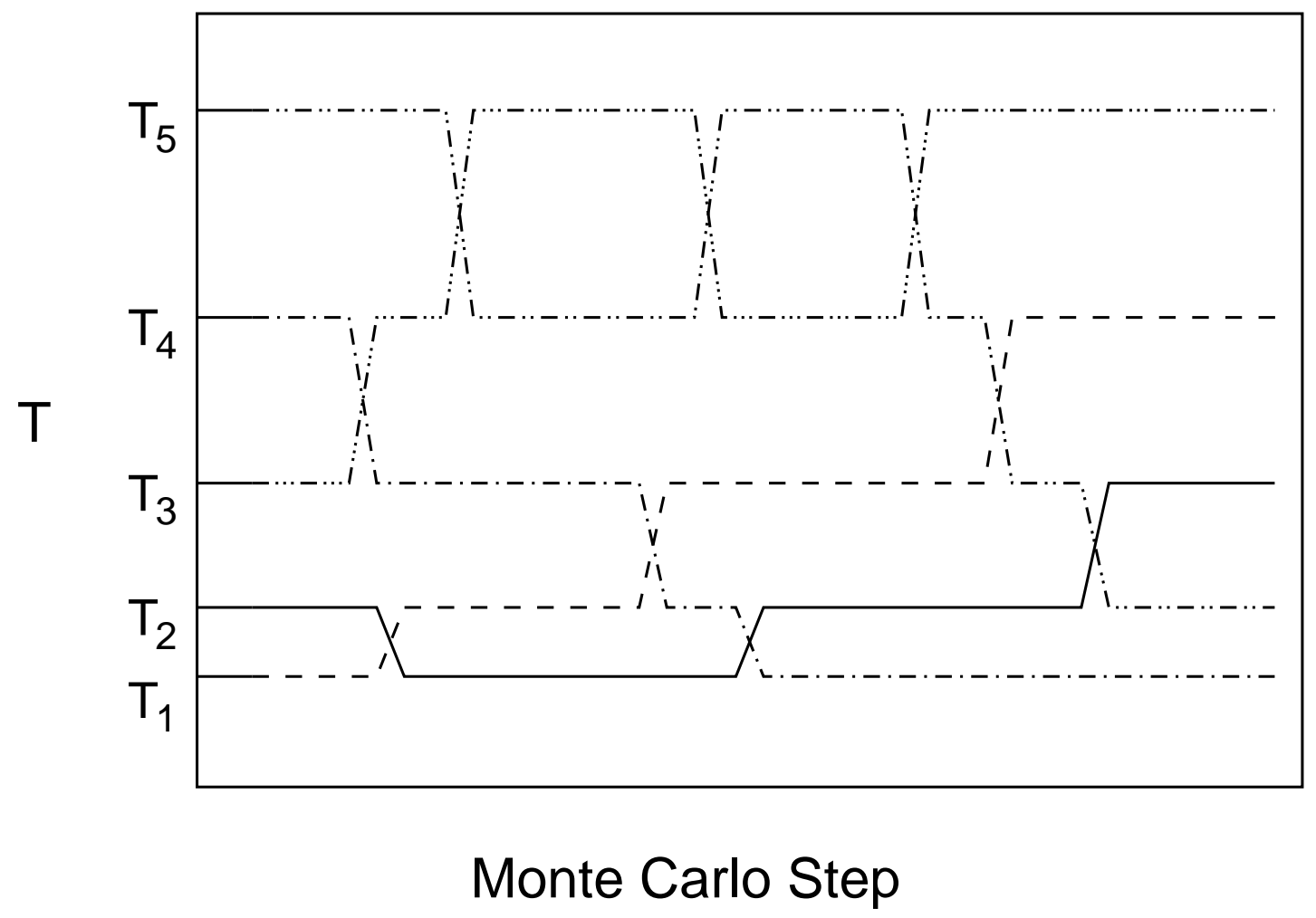

Figure 2: Schematic representation of parallel tempering swaps between adjacent replicas at different temperatures. In between the swaps, several constant-temperature Monte Carlo moves are performed. 


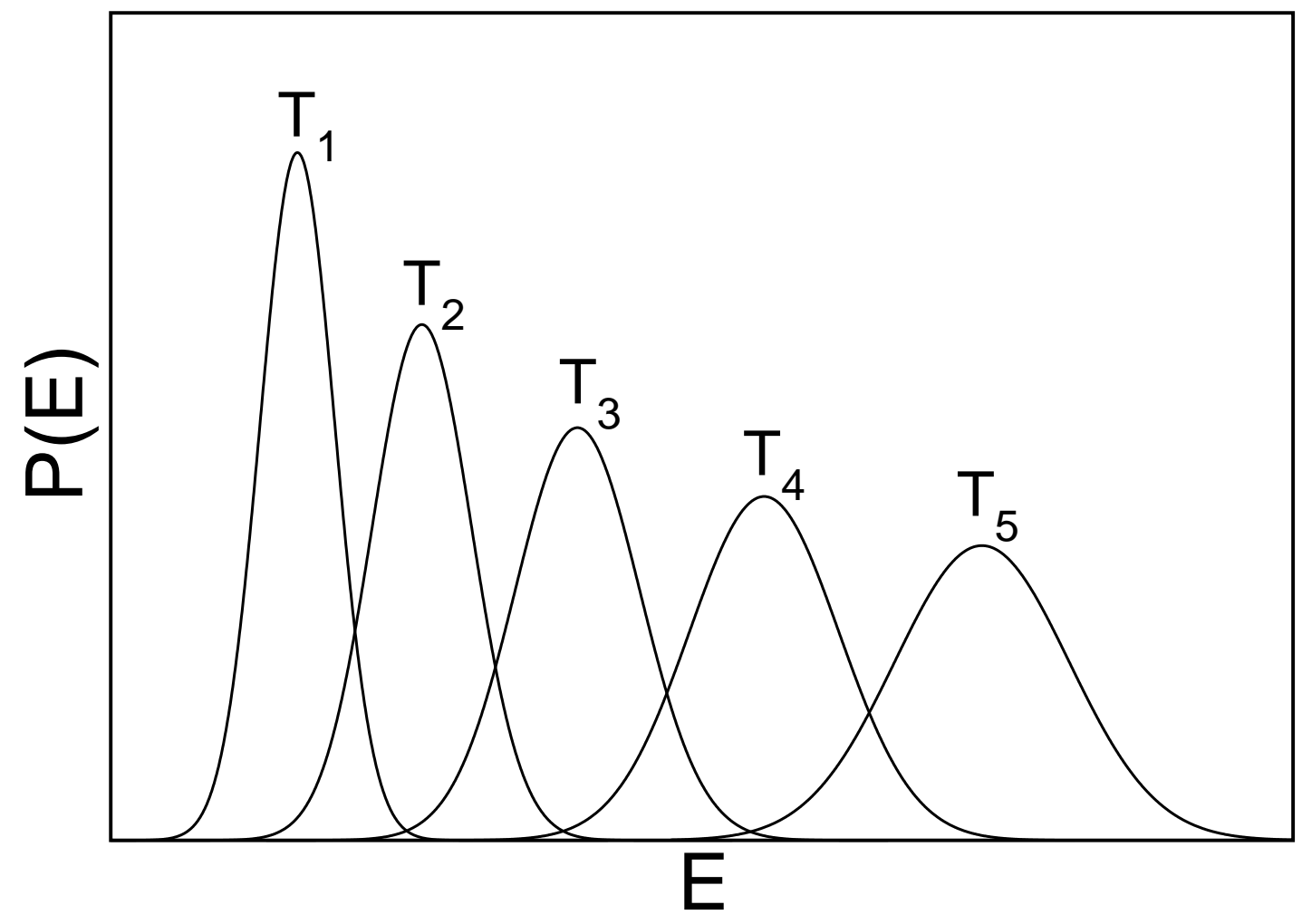

Figure 3: Energy histograms for a model system at five different temperatures. Overlap of the energy histograms between adjacent replicas at different temperatures allows for acceptance of the configuration swaps. 


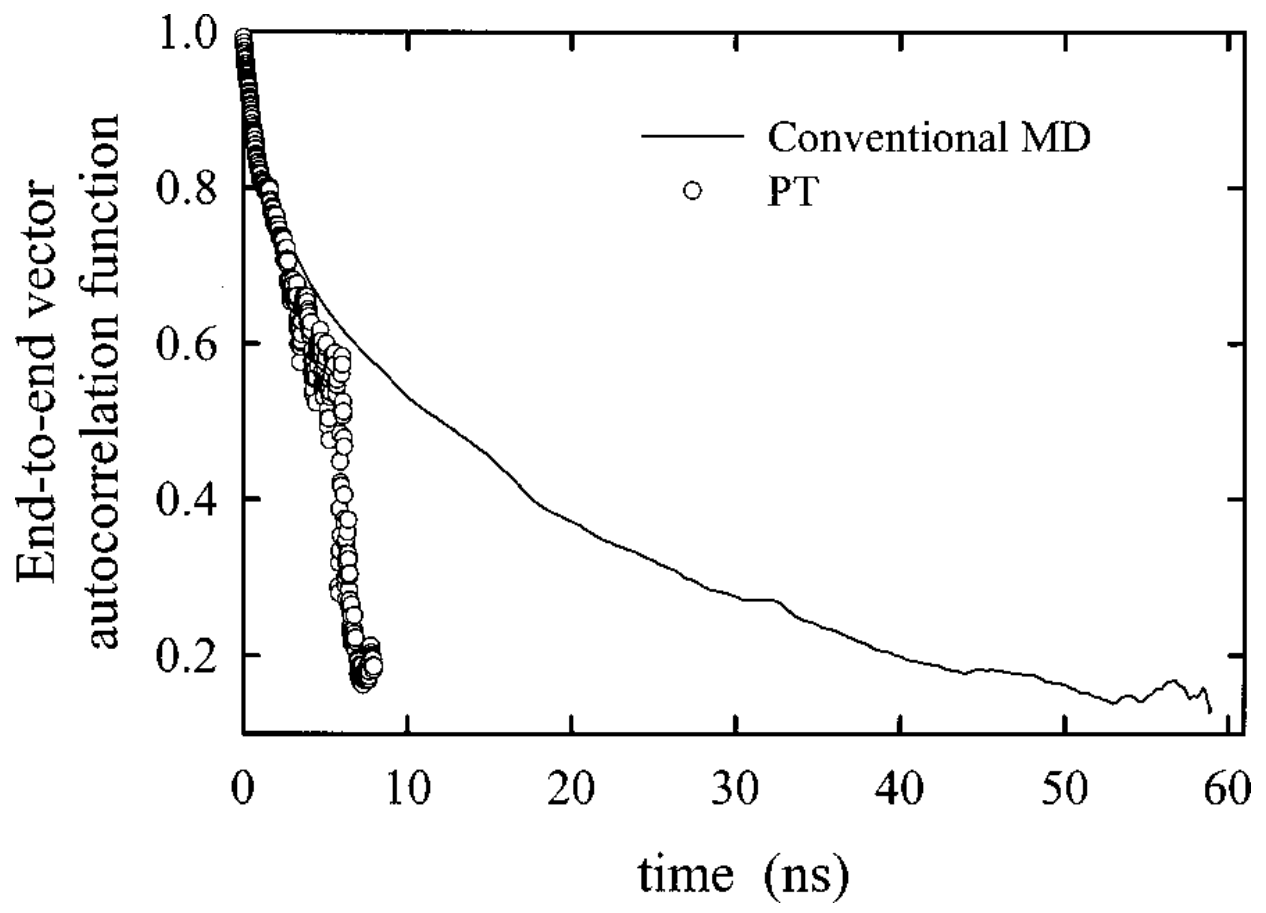

Figure 4: End-to-end correlation function for polymeric 1,4-polybutadiene. The parallel tempering simulation relaxes much more quickly, and is, thus, a more efficient simulation. Used with permission. ${ }^{45}$ Copyright 2001, American Institute of Physics. 